\title{
On Decomposing an Unseen 3D Face into Neutral Face and Expression Deformations
}

\author{
Faisal R. Al-Osaimi, Mohammed Bennamoun, and Ajmal Mian \\ The University of Western Australia \\ 35 Stirling Highway, Crawley, WA 6009, Australia \\ \{faisal, bennamou, ajmal\}@csse.uwa.edu.au
}

\begin{abstract}
This paper presents a technique for decomposing an unseen 3D face under any facial expression into an estimated 3D neutral face and expression deformations (the shape residue between the non-neutral and the estimated neutral 3D face). We show that this decomposition gives a robust facial expression classification and improves the accuracy of an off-the-shelf 3D face recognition system. The proposed decomposition system is a multistage data-driven process in which training expression residues and neutral faces reciprocally guide the decomposition of the 3D face. A plausible decomposition was achieved. The shapes and the normals of the expression residue are used for expression classification while the neutral face estimates are used for expression robust face recognition. Experiments were performed on a large number of non-neutral scans and significant expression classification rates were achieved. Moreover, $6 \%$ increase in face recognition rate was achieved for probes with severe facial expressions.
\end{abstract}

\section{Introduction}

Facial expressions are natural comprehensive means for communication that reflect the emotional states of humans. Automatic facial expression classification has significant potential in many applications especially in advanced humancomputer interaction. Similarly, face recognition has been the focus of research within the computer-vision community due to the immense need for accurate face recognition systems in many security applications such as surveillance and access control and also in human-computer interaction. Often both facial expression classification and face recognition are simultaneously required. For example, an intelligent robot system in a social environment requires a robust facial expression classification and a face recognition system.

The accuracy of facial expressions classification is adversely affected by variations in identity i.e neutral face variations among different people. Similarly, expression variations can affect the accuracy of face recognition systems. Decomposing a face into an estimated neutral face and an expression residue can assist in both expression classification and face recognition. We believe that there is more potential for robust face decomposition in the $3 \mathrm{D}$ domain compared to the 2D domain because of two reasons. Firstly, pose can be handled more robustly

M. Tistarelli and M.S. Nixon (Eds.): ICB 2009, LNCS 5558, pp. 22-31, 2009.

(C) Springer-Verlag Berlin Heidelberg 2009 
in 3D. Secondly, 2D images undergo affine transformations during the image formation and are also affected by illumination variations.

\subsection{Related Work}

Most of the existing expression classification [10 20 21/17] and face recognition [114 4 6] approaches attempt to improve their accuracies by extracting invariant features, identity-invariant for expression classification but expression-invariant for face recognition. Some systems assume the availability of neutral face images in case of expression classification (e.g. [15]) or assume that the probes and the gallery images are neutral in case of face recognition. Due to the complexity of the variations in facial expressions and the human face, these features are still believed to exhibit some sensitivities to these variations. To the best of our knowledge there is no existing work that decomposes a $3 \mathrm{D}$ or $2 \mathrm{D}$ face into a neutral face and an expression residue. Such a decomposition problem is relevant to expression synthesis which is addressed in 2D (e.g. 141993]) more thoroughly than in 3D (e.g. [16]). Usually, approaches to expression synthesis require prior knowledge of the identity and/or the initial expression (e.g. [1993]). In [122], a bilinear model which relates identities and a limited number of facial expressions (7 in their case) to Active Appearance Model (AAM) parameters is fitted to a training data using Singular Value Decomposition (SVD). During recognition, the bilinear model is used to find the identity and the expression corresponding to AAM parameters of a probe face by generating images under each of the considered expressions and matching them to the probe.

In a similar approach to [122], a tensor of order 3 is used instead of the bilinear model [18. These two approaches recognize simultaneously the identities and facial expression (unknown identities and expressions). However, these approaches are inherently incapable to generalize to unseen subjects and expressions as they can be considered as merely the resynthesis of the training expressions. The novelties in our decomposition approach are 1) our approach can decompose an unseen 3D face with unknown identity and expression; 2) it can handle an unlimited number of facial expressions (literally any deformations from the neutral face); 3) our approach has more potential for high accuracies since we utilize a large amount of training data of two types i.e. the neutral facial scans and accurate expression residues which were computed using ICP.

\subsection{Overview of the Proposed Algorithm}

Decomposing an unseen 3D face into an expression residue and a neutral face could be susceptible to the confusion between the neutral face and the expression residue shapes i.e. the neutral face estimate may include some expression related $3 \mathrm{D}$ geometries and similarly the expression residue may include neutral face related $3 \mathrm{D}$ geometries. Our system minimizes this possibility by employing a multi-stage decomposition approach. Initially, we project the unseen 3D face scan onto a PCA subspace which is computed from the shape residues between non-neutral and neutral 3D facial scan pairs (expression residues). These training 
scan pairs belong to many different people and the expressions of the non-neutral scans are diverse. However, each pair belongs to the same subject. We have demonstrated in our previous research [1] that the projection on such a subspace retains most of the expression deformations while it loses a large amount of the shape from the 3D face. In that work, we have shown that the projection can solve the expression deformation target problem, i.e. deforming a 3D facial scan to another 3D facial scan under different expressions, for the purpose of 3D face recognition. In this paper, there is no target $3 \mathrm{D}$ facial scan in the decomposition problem. Therefore, the projection alone is not enough. However, we considered it as a first stage in our decomposition system as it separates the unseen 3D facial scan into a part that is non-confusibly related to the neutral $3 \mathrm{D}$ face (the part which is lost in the projection) and another part in which confusion between expression and neutral face may occur (the part preserved during the projection on the subspace). This is because every point in the subspace does not necessarily represent a realistic expression residue.

In the second stage, the distributions of the training expression residues and neutral 3D scans in the expression residue subspace (i.e. the sets of their projection coefficient vectors) are utilized to guide the decomposition of the unseen 3D face projection. The expression residues and the neutral 3D scans belong to two different classes. The shapes of their distributions are expected to reside in the subspace taking different shapes. Starting from initial course estimates of the expression residue (equal to zero) and the neutral face (equals to the projection of the unseen 3D facial scan), these estimates are iteratively and reciprocally updated to the average of the nearest $M$ training residues and the nearest $N$ training neutral facial scans, respectively. Reciprocally here means that a change in either of the estimates also induces a counter change in the other estimate as the sum of the two estimates is constrained to be equal to the unseen face projection. When the search converges, the nearest $M$ expression residues and $N$ neutral scans are the best representations in the training data for the decomposed expression residues and neutral face projections.

In the last stage, the outcome of the second stage is further refined. In a fitting process the subspace around the expression residue estimate from stage two is searched such that the reconstruction of the expression residue plus each of the $N$ representing training neutral scans collectively fit the unseen 3D facial scan. This fitting is subject to an additional cost proportional to the deviation from the expression residue estimate from stage two. The result represents the final expression residue. Finally, the neutral face estimate is computed by subtracting the expression residue from the unseen $3 \mathrm{D}$ scan.

We also propose an expression classification approach based on the shape of the estimated residue and its normals. The normals are used to improve the classification of expressions with minor expression deformations because of their sensitivity to small surface deformations. The classification is based on fusion of expression labels of $k$-nearest expressions in four subspaces (one for the residue and three for $x, y$ and $z$ coordinates of the normals). The proposed classifier can handle combinations of fundamental expressions and also their extents. 


\section{Unseen 3D Face Decomposition}

The spikes in the 3D scans are removed by dropping the points that have statistically outlying $x, y$ or $z$ coordinates and the 3D surfaces at the holes are interpolated. The pointclouds are then decimated (for manageable computational complexity) and converted to a range image representation. The interpolation of the $z$ coordinates at integral $x$ and $y$ coordinates are stored in a matrix indexed by $x$ horizontally and $y$ vertically. Details of the 3D face decomposition are given in the following subsections.

\subsection{Stage 1: Projection onto PCA Residue Subspace}

The first stage in the decomposition of an unseen 3D face is the projection on a generic expression deformation PCA subspace. This PCA subspace is constructed from shape residues between training non-neutral and neutral scan pairs. The training scan pairs belong to a large number of subjects with diversity in gender and race. However, each individual scan pair belongs to the same subject. As a result, this PCA subspace largely represents expression deformations of the human face.

The preprocessed 3D scans are cropped and pose-corrected using the approach by Mian et al. [11. In that approach, the tip of the nose is first detected and the $3 \mathrm{D}$ points which are outside a sphere centered at the nose tip are dropped. The cropped 3D pointcloud is then aligned according to their principal directions. The self-occluded points appear as holes in the resulting pointcloud. The pointcloud is iteratively resampled and aligned until the pose stabilizes.

The pose-correction approach provides sufficient initial coarse registration for ICP and the localization of the semi-rigid regions of the 3D face by means of fixed binary masks (see Fig. 1.(a)). For the purpose of computing accurate shape residues, the 3D scan pairs are accurately registered according to the semi-rigid regions of the face (the nose and forehead) using the ICP algorithm [5]. The ICP algorithm is applied on the masked points (see Fig. 1(a)). A smaller mask is used for the non-neutral scan while a larger one is used for the neutral scan. Note that the second mask is a dilated version of the first one. This is to reduce the chance of inappropriate correspondences in the ICP algorithm by searching the pointcloud which is defined by the large mask for closest point correspondences in the pointcloud which is defined by the smaller mask. The rigid transformations which are calculated by the ICP are finally applied to the complete face. Fig.11(a) shows example scan pairs that were registered according to the semi-rigid regions of the face. The shape residue is computed for the $i$-th training scan pair as the difference between the non-neutral and the neutral scan, $\mathbf{R}_{i}=\mathbf{I}_{e_{i}}-\mathbf{I}_{n_{i}}$.

PCA $[\underline{8}$ is applied on the computed training residues. The residues are vectorized and the covariance matrix $\Omega=\sum_{i=1}^{n} \mathbf{r}_{\mathbf{i}} \mathbf{r}_{\mathbf{i}}^{\top}$ is then computed, where $\mathbf{r}_{\mathbf{i}}$ and $n$ are the $i$-th residue and the total number of the training residues, respectively. The eigenvectors of $\Omega$ that correspond to the top $k$ eigenvalues are computed. As opposed to using these eigenvectors to define an orthonormal lower subspace, we cropped these eigenvectors at the borders of the residues to remove the artifacts 
at the borders. This cropping is performed using a binary mask. The cropped eigenvectors span a non-orthonormal subspace. Eqn. 1 describes the projection of the unseen 3D facial scans onto such a subspace.

$$
\mathbf{c}_{i}=\left(\mathbf{E}^{\top} \mathbf{E}\right)^{-1} \mathbf{E}^{\top} \mathbf{f}_{i}
$$

where $\mathbf{f}_{i}$ is the projected face, $\mathbf{c}_{i}$ corresponds to the projection coefficients and the matrix $\mathbf{E}$ is formed from the cropped eigenvectors as column vectors.

The projection of a 3D facial scan described in Eqn. 1 loses a significant part when reconstructed using Eqn. 2, Whereas an expression residue is largely preserved during the same projection. This implies that the projection by itself significantly contributes to the decomposition process of the 3D facial scan. Further decomposition refinements are performed in the following stages (Section 2.2 and 2.3). Fig. 1.(b) shows example projections of 3D facial scans and shape residues on the expression residue subspace.

$$
\mathbf{r}_{p_{i}}=\mathbf{E} \mathbf{c}_{i}
$$
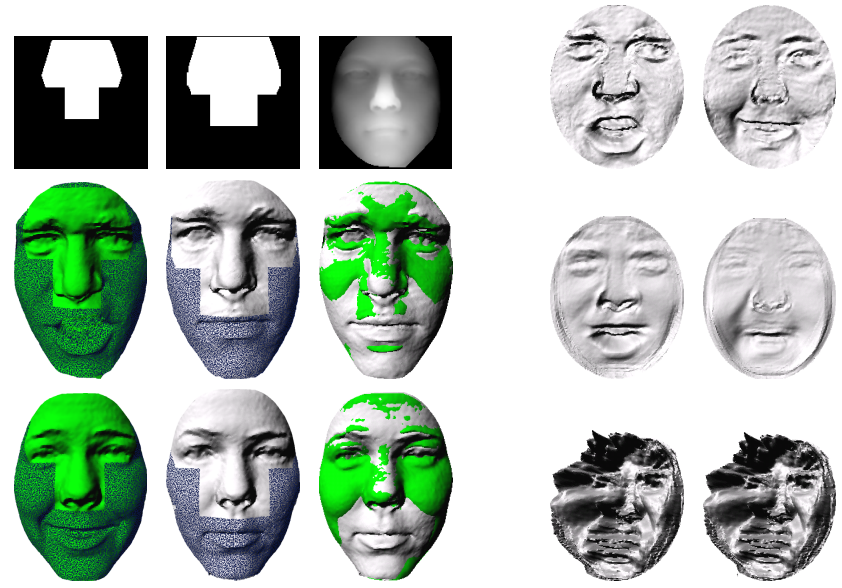

(a)

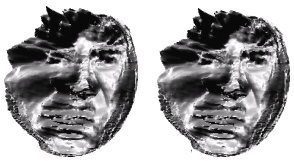

(b)

Fig. 1. (a) The binary masks (top row) which are used for scan pair (same subject) registration (second and third rows) according to the semi-rigid regions of the face using ICP. (b) The shapes of expressions residues (top row) are preserved during the projection on the PCA subspace (second row) whereas significant parts of 3D faces are lost during the same projection (third column).

\subsection{Stage 2: Iterative Estimation of the Residue and Neutral Face}

In this stage of the decomposition, the part of the $3 \mathrm{D}$ face which is preserved during the projection is further decomposed to expression and non-expression parts. The decomposition in this stage is data driven by training examples of $3 \mathrm{D}$ faces and expression residues. A large number of 3D faces and expression 
residues are projected onto the subspace using Eqn. 1] Let $\mathbf{f}, \mathbf{r}_{e}$ and $\mathbf{n}_{e}$ denote the projection coefficients of an unseen 3D face, expression residue estimate and neutral face estimate, respectively. Firstly, $\mathbf{r}_{e}$ is initialized to a vector of zero coefficients and $\mathbf{n}_{e}$ to $\mathbf{f}$. The $N$ nearest neutral face projection to $\mathbf{n}_{e}$ and the $M$ nearest neighbors of $\mathbf{r}_{e}$ are found, based on the city-block distance measure. $\mathbf{r}_{e}$ and $\mathbf{n}_{e}$ are updated using Eqns. 3 and 4. The process is repeated until convergence. The approach converges when the sets of nearest neighbors in an iteration are the same as in the previous iteration.

$$
\begin{aligned}
\mathbf{r}_{e_{i+1}} & :=\mathbf{r}_{e_{i}}+\frac{1}{2}\left(\overline{\mathbf{r}}_{i}-\mathbf{r}_{e_{i}}-\overline{\mathbf{n}}_{i}+\mathbf{n}_{e_{i}}\right) \\
\mathbf{n}_{e_{i+1}} & :=\mathbf{n}_{e_{i}}+\frac{1}{2}\left(\overline{\mathbf{n}}_{i}-\mathbf{n}_{e_{i}}-\overline{\mathbf{r}}_{i}+\mathbf{r}_{e_{i}}\right)
\end{aligned}
$$

The vectors $\overline{\mathbf{r}}_{i}$ and $\overline{\mathbf{n}}_{i}$ are the mean projection coefficients of the nearest $M$ residues and $N$ neutral faces at stage number $i$, respectively. Note that at any iteration the sum of the residue estimate and the neutral estimate is equal to the unseen 3D face, $\mathbf{r}_{e_{i}}+\mathbf{n}_{e_{i}}=\mathbf{r}_{e_{i-1}}+\mathbf{n}_{e_{i-1}}=\mathbf{f}$. The updating equations also ensure that at convergence the nearest neighbors simultaneously represent reasonable residue and neutral face estimates.

\subsection{Stage 3: Fitting to Nearest Neutral Faces}

In the final stage, the subspace around the residue estimate from stage 2 is searched for an estimate that collectively fits the difference between the input $3 \mathrm{D}$ face $\mathbf{f}$ and the nearest neighboring neutral faces $\mathbf{n}_{1 \ldots N}$ found in stage 2 at convergence. The fitting applies an extra cost proportional to the deviation from stage 2 estimate. A matrix $\mathbf{E}_{r}$ is formed by concatenation of $\mathbf{E} N$ times followed by a diagonal square matrix $\mathbf{M}, \mathbf{E}_{r}=\left[\mathbf{E}^{\top} \ldots \mathbf{E}^{\top} \mathbf{M}^{\top}\right]^{\top}$. The diagonal elements of $\mathbf{M}$ are $\left\{\frac{S}{\sigma_{1}} \ldots \frac{S}{\sigma_{k}}\right\}$. The factor $S$ determines the extent of the search portion of the subspace, a high value of $S$ implies that only small deviations from stage 2 solution are allowed. The differences between $\mathbf{f}$ and $\mathbf{n}_{1 \ldots N}$ are concatenated in a vector $\mathbf{r}_{r}$ followed by a search restricting vector, $\mathbf{r}_{r}=\left[\left(\mathbf{f}-\mathbf{n}_{1}\right)^{\top} \ldots(\mathbf{f}-\right.$ $\left.\left.\mathbf{n}_{N}\right)^{\top}\left(\mathbf{M r}_{e}\right)^{\top}\right]^{\top}$ where $\mathbf{r}_{e}$ is the residue estimate from stage 2 . The final residue estimate and neutral face estimate are expressed as in Eqn. 5] and 6. Fig. 2] shows example 3D faces and their residue and neutral face decompositions.

$$
\begin{aligned}
\mathbf{r} & =\mathbf{E}\left(\mathbf{E}_{r}^{\top} \mathbf{E}_{r}\right)^{-1} \mathbf{E}_{r}^{\top} \mathbf{r}_{r} \\
\mathbf{n} & =\mathbf{f}-\mathbf{r}
\end{aligned}
$$

\section{Facial Expression Classification and Detection}

Facial expression classification is performed based on the shape of the residue estimates and their normals. The normals are used because of their sensitivity to small shape variations which give the classifier a better ability to classify facial expressions that involve small surface deformations. The approach is to project 


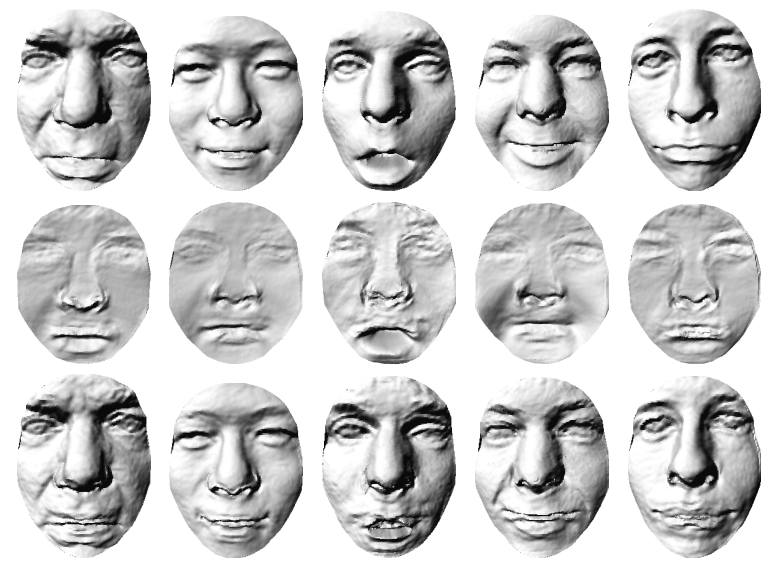

Fig. 2. Unseen facial scans (top row) decomposed into expression residues (middle row) and neutral face estimates (bottom row)

a large number of labeled training residues and the coordinates of their normals $(x, y$ and $z$ ) onto four corresponding PCA subspaces (one for the residues and three for their normals). The facial expression of the probe face is inferred from the labels of the $k$-nearest neighbors in each of the four PCA subspaces. We do not assume that individual facial expressions will form separate clusters in any of the four subspaces. However, we expect the labels to be locally consistent i.e. the labels of the nearby residues are similar but may be completely different for far away residues.

Six types of fundamental facial expressions were considered namely; happy, surprise, sad, angry, disgust and neutral. The expression classes can be one or a combination of these fundamental expressions leading to 26 classes in our case. Some combinations are not possible or did not appear in the training data. The labels also indicate the extent of the perceived expressions. An expression class is represented by a $6 \mathrm{D}$ binary vector with nonzero elements indicating the presence of the fundamental expressions in the class and zeros representing their absence. The value of the non-zero elements can be 1, 2 or 3 and they respectively represent major, moderate and minor expression levels. The labeling of the training data is is susceptible to human error which can affect its consistency and consequently affect the accuracy of the expression classification. To enhance the consistency of the manually labeled training data, we iteratively cross validate the labels by displaying the facial expressions and their $k$-nearest neighbors in a GUI interface and relabeling them if the labeling is not consistent.

For the inference of the expression class of an unseen probe face, a score vector of dimension 6 is computed from the $k$-nearest labels ( $k=10$ in our case) in each of the four subspaces resulting in a total of four score vectors $\mathbf{s}_{1 \ldots 4}$. A score of two is counted for each expression instance in the labels within the first five labels but only one score is counted for the last five labels. The expressions are detected by applying a vector of thresholds $\mathbf{t}$ to the score vectors $\mathbf{s}_{1 \ldots 4}$. The threshold vector 
$\mathbf{t}$ is initially set to large values $\mathbf{t}_{s}$ (a strong threshold vector). The expression scores which are more than their corresponding threshold in $\mathbf{t}_{s}$ are very likely to be present in the probe face. The detected expressions in the four subspaces may not agree with each other. To achieve better consensus between the results of the four subspaces, the strong thresholds are eased gradually until at least two of them achieve consensus subject to the condition that the resulting expression class is one of the predefined 26 expression classes. No further thresholding is performed if any element of $\mathbf{t}$ becomes less than its corresponding element in a minimum threshold vector $\mathbf{t}_{w}$ (weak thresholds). $\mathbf{t}$ is varied between the two threshold vectors by varying a parameter $\alpha$ from 0 to $1, \mathbf{t}=\mathbf{t}_{s}-\alpha\left(\mathbf{t}_{s}-\mathbf{t}_{w}\right)$. In the vast majority of cases, a consensus is achieved at this stage. However, if a consensus is not achieved, we threshold from the maximum possible scores towards $\mathbf{t}_{s}$ and search for consensus. If the consensus is still not achieved we take the first class apprears during the search for consensus (i.e the class which result from thresholding using the highest t). In all cases, we consider that a fundamental expression is detected if it appears in 3 or more subspaces when applying $\mathbf{t}_{w}$ and in addition to the previously detected expressions also forms a valid expression class. Fig. 3 shows examples of correctly classified expressions.

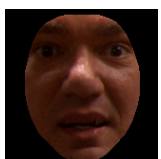

(a)

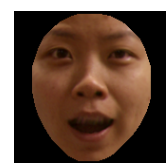

(b)

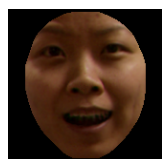

(c)

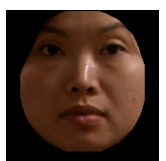

(d)

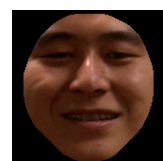

(e)

Fig. 3. Examples of correctly classified facial expressions. The expressions and their extents $\left(1,2\right.$, or 3 for severe, moderate and small, respectively) are surprise ${ }_{2}$ for (a), happy $_{3}+\operatorname{surprise}_{2}$ for (b), happy $2+\operatorname{surprise}_{2}$ for (c), neutral 1 for (d) and finally happy $_{2}+$ disgust $_{3}$ for (e).

\section{Experiments and Results}

The 3D face decomposition approach was trained and applied to unseen 3D faces (test data). Then the performance of the proposed expression classification approach and an off-the-shelf face recognition approach [8] were measured on the decomposed test data. These experiments require a large number of $3 \mathrm{D}$ facial scans for both training and testing which are mostly under non-neutral facial expressions. The FRGC dataset 13 was augmented in house by 503 3D facial scans acquired using a Minolta vivid scanner. The added scans belong to 3 subjects and 500 of them are under non-neutral expressions while only 3 scans are with a neutral expression (one per subject). From the FRGC dataset and the added scans a total number of 1948 residues and 262 neutral faces were used for training the decomposition approach and 1000 non-neutral scans were left for testing. The 1000 testing scans belong to different subjects (whose scans where not present in the training data). 
In the expression classification experiment, the non-neutral scans which were used to form the 1948 training residues were decomposed and their residue estimates were used for expression classification as described in Section 3. It is possible to use the training ground truth residues (difference between non-neutral and neutral scans) for classification rather than their estimates. However, experimental results have shown that the expressions are more accurately classified when the training residue estimates are used. Table 1 shows the results of the classifier. The detection rate of the fundamental expressions ranges from $96.22 \%$ to $83.02 \%$. High detection rates were achieved especially for happy, surprise and neutral expressions. The rate tangibly decreases for the sad, angry and disgust expressions. This decrease is generally attributed to the scarcity of representing training data for these expressions, the small differences between them and their high variabilities.

Table 1. Expression detection and false detection rates for six fundamental expressions. The classification rate represents the rate of detecting all the fundamental expressions that exist in an image without any false detections.

\begin{tabular}{|c|cccccc|}
\hline & happy surprise & sad & angry & disgust neutral \\
\hline Detection rate & $94.3 \%$ & $89.92 \%$ & $94.14 \%$ & $91.4 \%$ & $83.02 \%$ & $96.05 \%$ \\
False detection rate & $2.56 \%$ & $2.41 \%$ & $0.64 \%$ & $5.11 \%$ & $0.54 \%$ & $6.22 \%$ \\
\hline Classification rate & \multicolumn{5}{|c|}{$86.3 \%$} \\
\hline
\end{tabular}

The well-known PCA face recognition approach was applied to the decomposed neutral estimates. 406 estimates of neutral faces were extracted from nonneutral scans under severe facial expressions and used as probes. These probes were matched against a gallery of 100 subjects. The result was compared against the recognition performance when the decomposition was not used. The results shows about $6 \%$ increase in the identification performance when the proposed decomposition approach was used (39.9\% for the non-decomposed scans and $46 \%$ for their neutral face estimates).

\section{Conclusions}

This paper presented a novel approach for decomposing an unseen 3D facial scans under facial expressions to neutral face estimates and expression residues. The residues were used for expression classification and gave a high expression classification results. This paper also demonstrated that the decomposition approach can boost the performance of existing face recognition algorithms that are designed for neutral facial scans.

\section{References}

1. Al-Osaimi, F., Bennamoun, M., Mian, A.: An Expression Deformation Approach to Non-rigid 3D Face Recognition. IJCV (2009)

2. Abboud, B., Davoine, F.: Bilinear factorisation for facial expression analysis and synthesis. Vision, Image and Signal Processing (2005) 
3. Chandrasiri, N.P., Naemura, T., Harashima, H.: Interactive analysis and synthesis of facial expressions based on personal facial expression space. In: IEEE Conference on Automatic Face and Gesture Recognition (2004)

4. Chang, K., Bowyer, K., Flynn, P.: Adaptive Rigid Multi-region Selection for Handling Expression Variation in 3D Face Recognition. In: CVPR (2005)

5. Chen, Y., Medioni, G.: Object modeling by registration of multiple range images. In: ICRA (1991)

6. Chua, C., Han, F., Ho, Y.: 3D Human Face Recognition Using Point Signature. In: IEEE Conference on Automatic Face and Gesture Recognition (2000)

7. Faltemier, T., Bowyer, K., Flynn, P.: Using a Multi-Instance Enrollment Representation to Improve 3D Face Recognition. In: IEEE BTAS (2007)

8. Jollife, I.: Principal Component Analysis. Springer, Heidelberg (1986)

9. Lee, J., Lee, J.H., Kim, H., Moon, Y.: Automatic Synthesis of Realistic Facial Expressions. In: IEEE Symposium on Signal Processing and Information Technology (2007)

10. Matsugu, M., Mori, K., Mitari, Y., Kaneda, Y.: Subject independent facial expression recognition with robust face detection using a convolutional neural network. International Journal of Neural Networks (2003)

11. Mian, A., Bennamoun, M., Owens, R.: An Efficient Multimodal 2D-3D Hybrid Approach to Automatic Face Recognition. In: IEEE PAMI (2007)

12. Mpiperis, I., Malassiotis, S., Strintzis, M.: Bilinear Models for 3D Face and Facial Expression Recognition. IEEE Transactions on Information Forensics and Security (2008)

13. Phillips, P., Flynn, P., Scruggs, T., Bowyer, K., Chang, J., Hoffman, K., Marques, J., Min, J., Worek, W.: Overview of the Face Recognition Grand Challenge. In: CVPR (2005)

14. Ramachandran, M., Zhou, S., Jhalani, D., Chellappa, R.: A method for converting a smiling face to a neutral face with applications to face recognition. In: IEEE ICASSP (2005)

15. Ramanathan, S., Kassim, A., Venkatesh, Y., Wah, W.: Human Facial Expression Recognition using a 3D Morphable Model. In: IEEE Conference on Image Processing (2006)

16. Samper, S., Favreliere, H., Vacher, P., Coudert, T.: 3D measurement and synthesis of facial expressions. In: Symposium on 3D Analysis of Human Movement (2006)

17. Tang, H., Huang, T.: 3D facial expression recognition based on automatically selected features. In: IEEE Computer Vision and Pattern Recognition Workshops (2008)

18. Wang, H., Ahuja, N.: Facial Expression Decomposition. In: ICCV (2003)

19. Wang, S., Gu, X., Qin, H.: Automatic non-rigid registration of 3D dynamic data for facial expression synthesis and transfer. In: CVPR (2008)

20. Wang, J., Yin, L.: Static topographic modeling for facial expression recognition and analysis. Computer Vision ans Image Understanding (2007)

21. Zhan, Y., Ye, J., Niu, D., Cao, P.: Facial Expression Recognition Based on Gabor Wavelet Transformation and Elastic Templates Matching. In: International Conference on Image and Graphics (2004) 